\title{
The Open Rehabilitation Journal
}

Disclaimer: This article has been published immediately upon acceptance (by the Editors of the journal) as a provisional PDF from the revised version submitted by the authors(s). The final PDF version of this article will be available from the journal URL shortly after approval of the proofs by authors(s) and publisher.

\section{Memory Impairments and Rehabilitation: Evidence-Based Effects of Approaches and Training Programs}

Olimpia Pino

The Open Rehabilitation Journal, Volume 8, 2015

ISSN: 1874-9437

DOI: $10.2174 / 1874943720150601 \mathrm{E} 001$

\section{Article Type:}

Received: November 10, 2014

Revised: $\quad$ May 15, 2015

Accepted: $\quad$ May 25, 2015

Provisional PDF Publication Date: June 03, 2015

C Olimpia Pino; Licensee Bentham Open.

This is an open access article licensed under the terms of the Creative Commons Attribution Non-Commercial License (http://creativecommons.org/licenses/by-nc/3.0/) which permits unrestricted, non-commercial use, distribution and reproduction in any medium, provided the work is properly cited. 
Memory Impairments and Rehabilitation: Evidence-Based Effects of Approaches and Training Programs

\author{
Olimpia Pino \\ Department of Neurosciences \\ University of Parma, \\ Piazzale S. Francesco n. 2, 43121 - Parma (Italy) \\ E.mail:(olimpia.pino@unipr.it)
}

\begin{abstract}
This paper provides an update and overview of the field of memory disorders and memory rehabilitation of adults. Progresses in brain plasticity knowledge and neuroimaging observations about compensation after structural losses have influenced non-pharmacological interventions, which represent a growing area with strong theories and explanatory frameworks.

Rehabilitation models may follow three directives not alternatives to each other: restore lost functions, develop compensatory strategies or adopt external memory aids. Because of treatment approaches have a differential effect on distinct aspects of memory functions, and the assessment of the treatment outcomes is relevant not only at impairment level but also at the disability level, the effectiveness of restitution oriented therapies and compensatory approaches was evaluated. We discussed these different treatment approaches also reporting a survey of training results and systematic reviews findings. A large body of evidence seems to support, with different efficacy levels according to etiology and type of brain injury, several training programs in stable individuals but not in persons with degenerative disease. The present paper can serve as a guide for clinicians and researchers.
\end{abstract}

Keywords Amnesia, Brain stimulation, Memory, Memory disorders, Memory intervention, Memory trainings.

\title{
1. Introduction
}

Memory declines linearly along the life span with aging, but it is in particular conditions such as Mild Cognitive Impairment (MCl), dementia, or other clinical disorders, such as traumatic brain injury (TBI) or stroke that memory deficits are dramatically manifest. Recent advances in cognitive neurosciences have clarified many neural networks involved in typical memory functioning and elucidated why focal injuries or degenerative disorders involve particular memory dissociations and deficits. Individuals with brain injuries are unfortunate natural experiments that have allowed us to investigate these deficits accumulating a knowledge base on the neurophysiological and functional organization of memory [1]. Brain injuries often cause severe and unpredictable deficits suggesting indications about functional networks involved in memory functions. Typically, the neural basis of training-related performance gains refers to patterns of functional changes in localized brain regions engaged by the training target.

Memory impairments have a devastating effect on the psychological wellbeing of the individuals and others around them. The safety of such patients can be compromised making them at risk citizens in their home, community, and work situations. The increasing attention for cognitive rehabilitation and neuropsychological interventions for memory deficits is justified by two main reasons: a) the poor outcomes obtained with pharmacological treatments, b) the advancement in 
brain plasticity knowledge. Memory intervention techniques focus on maximizing individuals' everyday functioning and can be summarized in three main approaches:

a) Restore lost functions stimulating the adoption of appropriate encoding and retrieval strategies; restorative interventions targeted to aid a return to the pre-injury condition. Techniques frequently used are those in cognitive rehabilitation and explicit learning and memory. Internal strategies involve training on the mental manipulations aimed to simplify memory of target stimuli. For example, a person may group ten items needed at the grocery store into categories (i.e. semantic organization or categorization). The more an individual's targeted strategies are functional or applicable to everyday life, the more likely they be applied.

b) Develop compensatory strategies based on spared functions; compensatory interventions aim to support people "work around" difficulties by finding new ways to accomplish tasks. The use of implicit and procedural knowledge involved into techniques that not require consciousness is a way to complete several tasks.

c) Adopt external memory aids. External compensatory strategies involve some form of assistance outside oneself, for example, a memory notebook to remember the day's activities.

Figure 1 summarizes the most common memory rehabilitation techniques grouped according to their principal "mechanism of action." The selection of a specific approach depends on the assessment process including type of brain injuries, impairment levels, spared functions, memory strategies reports and current needs. Given findings regarding injury severity and the close relation between strategic aspects of memory and executive functions, a recent study suggested that levels of memory impairment are the best predictor of outcome for cognitive rehabilitation in a wide range of conditions [2].

\section{Memory Rehabilitation Techniques}

\begin{tabular}{|l|}
\hline Implicit Training \\
\hline - Spaced Retrieval \\
- Vanishing cues \\
- Procedural learning \\
- Implicit memory \\
\\
\hline
\end{tabular}

\begin{tabular}{|l|}
\hline Mnemonic Strategies \\
\hline - Rehearsal \\
- Semantic \\
organization \\
- Semantic elaboration \\
- Imagery \\
- Method of Loci \\
- Computer training \\
programs/games \\
\hline
\end{tabular}

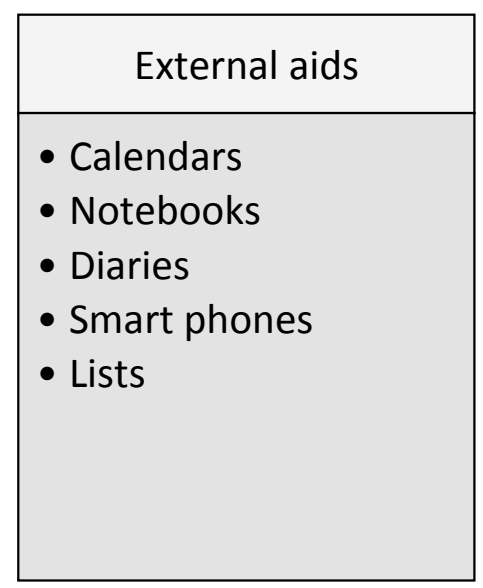

Figure 1. Classification of memory rehabilitation techniques based on their action mechanism The aim of the present paper is to describe the different approaches to memory rehabilitation for several disorders in adults considering evidence-based recommendations. Although variability in the diagnostic criteria used, techniques investigated, training amount, and outcome measures make a coherent conclusion difficult, rehabilitation approaches aimed to restore lost functions, develop compensatory strategies or adopt external memory aids will be discussed together with their effect on memory functions in different disorders and outcome quality.

\section{The case of memory impairments due to aging: Evidence for a compensatory mechanism.}

With aging learning, remembering and attention processes decline: for older adults episodic tasks [3], which involve encoding of current experiences and/or retrieving through temporal and spatial 
cues, resulted particularly problematic. Changes in episodic but also in the semantic components of memory reflect the age-related brain modifications, which undergo noteworthy structural and functional alterations with aging, typically attributed to reduced cell metabolism, which is characterized by a decrease in the activity of networks devoted to peculiar functions.

Demographic aging is the main factor involved in the raising of the mean age of the general population and the corresponding increase in the frequency of cases that develop neurogenerative age-related diseases. Consequently, in order to reduce the impact of these deficits in older adults, it was necessary to identify effective interventions. An intriguing discovery was that for several older persons it is possible to balance the structural impairments of aging showing task performances parallel to those of younger individuals or healthy older people. Therefore, the investigation of why and how these different age-related cognitive differences occur is of extremely significant attention. Tulving and colleagues proposed a rectification of the Hemispheric Encoding Retrieval Asymmetry (HERA) model [4] for older individuals, based on the neural mechanisms underlying age-related modifications. HERA is a general framework for brain functioning sub-serving episodic memory conceptualized based on prefrontal activation during encoding-retrieval tasks, as observed through neuroimaging and TMS investigations. It suggests a specialization of the left prefrontal cortex (PFC) for encoding activity and the right PFC for retrieval. Healthy elders show a bilateral engagement of the PFC in both encoding and retrieval, different from that evidenced by young subjects - who showed only left hemisphere activity during retrieval (although the activation of the right PFC during recall was less noticeable). The implications of these modifications are fascinating because they could represent either a functional compensation mechanism or a less effective processing capacity for the contralateral hemisphere. This finding led to the specification of the Hemispheric Asymmetry Reduction in OLDer (HAROLD) framework [5].

During the last few years repetitive transcranial magnetic stimulation (rTMS) has been utilized to study prefrontal asymmetry in healthy older persons, thereby confirming the reduction in asymmetry, and showing that older adults with better memory functioning presented little prefrontal asymmetry [6]. This discovery suggests the possibility that decreased asymmetry could represent an efficient strategy related to the brain plasticity and cognitive reserves for counteracting age-related decline [7].

\section{An overview of treatment approaches in memory disorders}

Non-pharmacological rehabilitation of memory impairments in healthy older adults, but particularly in individuals with brain injury and neuro-degenerative disorders, has received a great deal of interest. Clinical practice guidelines on cognitive rehabilitation suggest that treatment approaches have a differential effect on distinct aspects of memory functions. On the other hand, the importance of assessing the treatment outcomes not only at impairment level but also at the disability level was also stressed. Consequently, the effectiveness of both restitution-oriented therapies and compensatory approaches was evaluated [8]. The holistic neuro-psychological rehabilitation framework is an extremely individualized approach, recognizing the non-uniformity of structural alterations, personal conditions and copying strategies. The cognitive treatment approach initially addressed traumatic brain injuries but then it was extended for treating degenerative disorders such Alzheimer Disease (AD) or other forms of dementia at any severity stage, focusing on treating distinct emergencies during the course of the disease, later evolving into a palliative management towards the end of life [9-10]. Training programs aimed to tackle the impairments indicated as most significant by individuals and their caregivers, accordingly in particular target areas where memory affects everyday functioning. The use of cognitive rehabilitation during early dementia is particularly relevant considering the neuro-psychological patterns of persons with $A D$, which show severe short-term and explicit memory impairments whereas long-term recall and implicit memory were relatively uninfluenced. These individuals show significant deficits particularly in episodic memories evident in free recall or recognition tasks. Plasticity mechanisms also have an effect in AD showing a gain in the activation of areas participating in memory, or in a recruitment of new regions. 
In the next paragraphs will be summarized the results of three previous reviews and metaanalytic study $[7-8,11]$ adding nineteen investigations. We refer interested readers to the previous reviews for a thorough discussion and more details of the subject.

In order to suggest evidence-based recommendations, Piras and colleagues [8] systematically assessed the papers containing re-training programs, external memory aids and domain-specific learning strategies published between 2003 and 2007. Their conclusions are that re-training and compensatory interventions are "probably" effective for treatment of memory disorders in individuals with focal brain lesions, whereas the use of assistive devices and strategies is valuable for memory in everyday activities also for persons with degenerative diseases. However, the evidence about the effectiveness, extension and length of non-pharmacology rehabilitation outcomes is limited. A recent review [7] reports a summary of programs targeted on memory training for Mild Cognitive Impairment (MCl) and $A D$ individuals focusing specifically on cognitive techniques and non- invasive brain stimulation (e.g.: rTMS and tDCS). In the following paragraphs, I will describe the different approaches to memory rehabilitation.

\subsection{Function recovery and re-training programs}

Cognitive rehabilitation is a structured set of therapeutic activities aimed to retrain an individual's memory and other cognitive functions. The cognitive rehabilitation approach is centered on explicit mnemonic training concerning the use of imagination, organization strategies and techniques and it is based on the prediction that strategies will successfully maintain the levels required for everyday functioning. Among these "internal" techniques aimed at optimizing encoding and/or retrieval skills, the most typically used and studied were verbal elaboration and visual imagery [10]. Some randomized controlled trials (RCTs) have showed the effectiveness of cognitive rehabilitation but most of this evidence comes from single-case design studies and controlled clinical trials [2, 8, 11-15].

The effectiveness of re-training programs seems to depend on etiology and type of brain injury; in fact, training programs are effective only in stable individuals, not in persons with degenerative disease. At the outcome level, improvements extended to untrained skills affecting memory functioning in everyday activities occurred only in a small percentage of cases.

A crucial differentiation was that emphasized by the World Health Organization model of disability between structural alterations and impairments in everyday functioning and/or social participation, since personal engagement in daily activities and social participation are affected by personal and social factors and not directly by organic disease. The main purpose of cognitive rehabilitation is to enable individuals with functional diseases to reach the best possible level of well-being, decreasing disabilities (limitations on engaging in activity) and handicaps (limitation in social participation), consequences of traumatic brain injury or degenerative disorders. A second purpose was management of risks and other conditions originating from secondary reactions to the situation, such as depressive symptoms or decrease in self-esteem.

Several studies on individuals with neurological disorders have indicated that progress in the recovery of functions after traumatic brain injury or other non-progressive disorders could involve a reorganization of the brain networks after rehabilitation interventions. A RCT $(n=72)$ was carried out to assess a group memory rehabilitation program [14]. Patients with memory deficits are randomly assigned to one of three group treatments: 1) compensation strategy training, 2) restitution, or 3 ) a self-help attention placebo. Training programs include restitution strategies devoted to retraining of memory functions, attention retraining and strategies to improve encoding and retrieval. Compensation strategies are taught, including internal mnemonics (chunking, first letter cues, rhymes), use of external devices (mobile phones, calendars), and ways of coping with memory deficits. Data showed that there were no statistically significant differences in outcome. Currently, TBI subjects with memory problems are not routinely involved in memory rehabilitation after the early intensive phase. This is mainly due to the current lack of evidence of clinical and cost-effectiveness of the intervention, and resource limitations.

The main purpose of a second multi-center study [16] is to ascertain whether adding memory training in TBI subject groups show subjective reports of improved memory in daily life when compared to usual care (UC) control. Study is still in progress. A third study [17] aimed to 
evaluate the effects of involvement in a memory group trial focusing on Internal Memory Strategies (I-MEMS) with ninety-four adults with TBI (from mild to severe). This study compared two training methods: 1) error-free learning, whereby patients were first provided with cues in order to ensure the correct performance, 2) metacognitive strategy training, whereby subjects learned to assess both task-specific memory demands and their general memory ability, and then used these strategies to maximize their performance. Results indicated that the participation in the memory group caused an improvement in memory, and that these results were maintained at the follow-up (1 month). Severe injury was associated with less progress in outcome measures than mild and moderate injuries.

In the last few years, the tendency to focus on prevention is common, especially with specific elderly populations with $\mathrm{MCl}$ or amnesic $\mathrm{MCl}(\mathrm{aMCl})[18] . \mathrm{MCl}$ is a classification that include a range of age-related cognitive impairments in individuals who eventually show a dementia prodrome condition, and it defines a disorder with subjective cognitive alterations, objective memory impairments, or other cognitive deficiencies without deterioration in everyday functioning. The National Institute on Aging (NIA) of the National Institutes of Health in the United States, in collaboration with the Alzheimer's Association (AA) define AD as a progression with three principal stages: (i) asymptomatic, or preclinical; (ii) prodromal symptomatic, or mild cognitive impairment (MCl) due to AD; (iii) fully symptomatic, or AD dementia [19]. Given the enhanced risk of developing $\mathrm{AD}$ for $\mathrm{MCl}$ individuals, there are robust motivations for the design of nonpharmacological treatments targeted to decrease memory impairments in $\mathrm{MCl}$ persons. This continuum certainly presents a challenge for cognitive rehabilitation and entails careful selection of outcome measures that are widely applicable. Neuropsychological predictors of conversion encompass performance on delayed recall and executive functions. Reducing or recovering from recognized risk factors is important in the prevention of the conversion from $\mathrm{MCl}$ to $\mathrm{AD}$ or to delay the progression of symptoms.

A randomized trial [20] was carried out in order to test whether 12 months of supervised multicomponent exercise improved cognitive function among older adults with aMCl. The multicomponent exercise included aerobic exercise, muscle strength training, and postural balance retraining under the supervision of physiotherapists for $90 \mathrm{~min} / \mathrm{d}, 2 \mathrm{~d} / \mathrm{wk}$, for a total of 80 times over 1 year. The subjects practiced aerobic exercise, postural balance retraining, and dualtask training for $60 \mathrm{~min}$; for example, during an outdoor walk participants were asked to invent their own poem while walking or memorize a step pattern in segments of consecutive squares. The participants were requested both to record the amount of time spent on daily home-based exercise and the daily total steps on the pedometer in a notebook and made graphs of the results to promote active lifestyle through a self-monitoring. Positive effects were shown for general cognitive function, immediate memory, and verbal fluency although the effects on delayed memory, processing speed, and executive control did not have statistical significance. Enhancing cognitive function in $\mathrm{MCl}$ may prevent conversion from $\mathrm{MCl}$ to $\mathrm{AD}$ in older adults.

Several studies have evaluated the results of memory training on individuals with $\mathrm{MCl}$, showing prospective memory [21], quality of life and meta-memory [12] increases. In order to analyze brain activity changes as result of cognitive training in $\mathrm{MCl}$ subjects, several investigations include functional imaging techniques. An fMRI study [22] showed the validity of explicit memory training in individuals with $\mathrm{MCl}$ multidomain through the demonstration of specific raises in both activation and connectivity across networks implied in explicit memory process. This and other investigations provide strong evidence for the application of cognitive programs as treatment for $\mathrm{MCl}$ persons, although the great heterogeneity of this group prevents a full generalization. Cipriani et al. [23] evaluated a computer-based program involving activities designed to train both acquisition of new information and recall from semantic memory in $A D$ and $\mathrm{MCl}$ persons, and in a control group of subjects diagnosed with Multiple System Atrophy (MSA). Results indicated that the $\mathrm{MCl}$ participants significantly improved their working memory, psychomotor learning, and behavioral memory while the AD individuals developed their cognitive status (MMSE), verbal fluency and executive functions indicating a beneficial training effect also in the cognitive tests. Variability in the rate of reversion from $\mathrm{MCl}$ to typical functioning was found, that depended on the diagnostic criteria and psychometric cutoff values used. Reversion is more probable when subjects are less impaired, as defined either by the number of memory tests showing decreased performance or by the level of psychometric impairment. 
Prospective memory (PM) deficits are more usual among persons with traumatic brain injury (TBI) than controls [2]. TBI typically concerns accidents to the prefrontal cortex related to executive functions. Prospective memory requires a chain of executive processes, such as planning, disregarding of an ongoing activity, beginning of the scheduled action, and strategy adoption. It is not surprising thus, that deficit in executive functions has been shown to predict PM impairments. Treatments aimed to increase memory efficiency are an essential characteristic of rehabilitation programs after TBI in order to improve independence and community reintegration, and also because memory impairments are one of the most typical disorders of brain injured persons [13]. Prospective memory (PM) or the execution of delayed intentions is a crucial skill for success in everyday functioning [24].

Prospective memory functions in order to realize composite goal-directed actions and enable an individual to accomplish their plans in a purposeful sequence and at the correct time. Subjects who have lost this capability reveal the importance of PM in everyday activities. There is strong evidence that some important processes for fulfilling delayed intentions are based on brain networks placed in the frontal lobes and related areas. Although the employment of a compensatory approach is common in most brain injury training, a small number of investigations have explored compensatory aids to PM treatment.

A key factor for the verification of Shum et al's claim [25] is the individuals' awareness of the extent of their memory deficits. Nevertheless, self-consciousness is a higher cognitive function related to prefrontal cortex functioning, usually impaired following TBI. The purpose of this investigation was to assess the role of self-awareness training and compensatory programs on PM in groups of participants with moderate-severe TBI. Results showed large improvements in PM test rating and strategy use for subjects using the compensatory prospective memory program suggesting that it is possible to reinforce prospective skill in individuals suffering from traumatic brain injury with the experimental model. However, their results did not confirm the efficacy of the treatment package involving self-awareness and compensatory activity. Generally, cognitive training was associated with a pattern of activation decrease across a broader network of brain regions. Indeed, post-training decreases in activation are consistently considered as a neural marker for well-automated or highly trained performance.

\subsection{Memory rehabilitation procedures based on non-declarative knowledge}

The second approach addresses knowledge learning in specific domains whose purpose is the teaching of essential information and skills to amnesic patients to support their everyday functioning. Usually these interventions focus on non-declarative knowledge as in procedural memory training. Procedural memory defines the effect of previous experiences on current behavior without the occurrence of conscious recognition. AD patients demonstrate the capacity to retain it [26]. Stimulation of procedural memory induces an improvement in everyday functioning so that the positive results from this treatment were commonly restricted to the program period and there was a rapid decline of these gains after the completion of training. Treatment of individuals with strong memory deficits is extremely demanding for researchers in clinical settings. A common cognitive approach used in AD rehabilitation includes errorless learning techniques. Neuro-psychological evaluations have indicated that, particularly during the first phases of the disorder, relatively well-preserved skills should be the target of rehabilitation with the aim of delaying cognitive functioning impairment. Studies using errorless learning techniques for individuals with $\mathrm{MCl}$ indicate that this method can aid information acquisition and the associations' constitution useful for daily life.

Errorless techniques indicate learning situations in which error is excludes. Baddeley and Wilson [27] showed their effectiveness with amnesic individuals. The procedures of errorless training techniques include the method of vanishing cues (MVC) and the spaced retrieval (SR) [28-29]. Sufficient scientific evidence supports the use of EL over errorful techniques. Because recurrent priming may create lasting memory traces, that may sustain long-term retention of declarative memory, impaired individuals are able, if provided with fragments to attain procedural and novel semantic information of a factual nature. MVC is effective in learning domain specific information, although access to this information occurs only in particular contexts and not in transfer tasks. 
The vanishing cues approach requires the presentation and consequent progressive diminution of cues that suggest correct responses across learning trials. Spaced retrieval concerns the request for target recall over increasingly longer time intervals whereas the technique of subtracting cues gradually removes aspects of the target information (e.g., the letters of a name) over successive presentations. Rehearsal can be time consuming and even difficult (e.g., with spaced retrieval), factors that may further limit the clinical utility of these procedures. Although many available computerized programs claim to improve cognitive abilities and there is one report of increased hippocampal activation after training, such approaches have typically produced conflicting evidence of generalization to standardized neuropsychological tests or everyday functioning across patient populations [15].

Baddeley and Wilson [27] criticized the vanishing cues method in that it promotes error occurrence, particularly in the first learning sessions. They claim that a relevant function of explicit memory during acquisition is to suggest and prevent errors: if explicit memory declines (as in dementia), learning of new information may be faulty due to the interference of previous errors stored in implicit memory. They demonstrated that errorful procedures discontinued the learning of memory-impaired participants, in comparison with younger or older healthy subject's performances. The errorless paradigm involves both faster learning and less forgetting of items as it restricts the possibility of incurring errors because individuals receive the exact response rather than the request to guess or explicitly recall it.

Imagery is the most well evaluated mnemonic method. Leng and Parkin [30] show that amnesic individuals profit from mental imagery in an unsophisticated word association task. The imagery program can be organized into a skill-acquisition phase, in which participants are trained to apply imagery in progressive demanding conditions (e.g., imaging of single items, several items or actions, while the retrieval intervals were gradually extended and a transfer phase, in which individuals practice mental imagery according to their individual needs. Because subjects with memory impairments are often surprised that they are able to give correct responses without conscious recollection of previously presented information [31], it seems that the method functions without cognitive demand. Therefore, the effectiveness of spaced retrieval appears related to implicit knowledge.

The spaced-retrieval procedure prompts the remembering of target over progressively longer retention intervals. This procedure derives from the finding that massed practice is an inferior effective learning method to distributed practice [32]. Schacter and colleagues [31] were the first to demonstrate that spaced retrieval may be useful in amnesic individuals. This system was later applied to the treatment of memory impairments in Alzheimer's disorder. Sohlberg and Mateer [33] formulated a prospective memory training system based on spaced retrieval, with the aim of improving recall of scheduled tasks and appointments. Training required subjects to complete simple tasks in a specified time, progressively increasing the intervals. Results indicated a gradual increase in the prospective memory span in individuals with acquired brain injury. Some authors referred to spaced retrieval as a shaping procedure where target long-term retention is broken into sub-steps that are gradually more demanding until the individual shows recall in everyday conditions. This technique involves the procedural knowledge active in stimulus-response conditioning which response is reinforced by its specific consequence; it is very similar to the time-delay procedure of stimulus control transfer [34]. Although several studies indicated SR as potentially effective in treatments of memory disorders from different etiologies, only one study [36] addressed maintenance after training discontinuation. Therefore, only an EL approach can be recommended as a practice guideline for individuals with acquired memory disorders consequent to a stable or progressive disease.

Individuals with frontal lesions shown abnormalities in the "strategic" aspects of memory, which are closely tied to executive functioning and the prefrontal cortex. In these cases, manipulation of information that normally facilitates subsequent encoding, storage, and retrieval of that information is disturbed. In TBI pathologies it was also reported a functional breakdown in connectivity between left dorsolateral prefrontal cortex and other regions when individuals tried to learn word lists using semantic encoding strategies. Although we cannot rule out the possibility that individuals with TBI may be potential candidates for internal memory strategy training, evidence suggests with strongest support for training that emphasizes external memory strategies. 
Cognitive deficits in dementia may result from a decreased blood flow ( $\mathrm{rCBF}$ ) in the frontal, parietal, and temporal association cortices. In a pilot study, [Kawa 2005] authors hypothesized that activation of the association cortices principally the dorsolateral prefrontal cortex by cognitive tasks may improve rCBF and metabolism of these cortices leading to improvement in the function of these cortices. Daily Learning Therapy program over more than 6 months included two tasks in arithmetic and reading problems in Japanese. Reading aloud requires a combination of several cognitive processes such as recognition of visually presented words, conversion to phonological representation from visual pattern of words, analysis of their meaning, and control of pronunciation. Solving arithmetic problems requires various cognitive processes such as recognition of visually presented numbers, arithmetic operations, and control of hand movements. Additionally, the bilateral prefrontal cortices are active even during the solution of simple problems. Both reading aloud and solving arithmetic problems require working memory. Cognitive ability measured by MMSE did not decline during the 6-month learning period. Learning Therapy is different from previous systems of cognitive rehabilitation. First, this program is not evidencebased because it relies on the theoretical background of the knowledge of neuroscience research - that is, solving arithmetic problems and reading aloud activate bilateral dorsolateral prefrontal cortex. In addition, it aims to facilitate transfer of the different cognitive functions within the dorsolateral prefrontal cortex - from reading aloud and solving arithmetic problem functions to general cognitive functions such as communication and independence. Finally, distinguishing whether the benefits for the experimental group depended on the cognitive training itself or on interaction with the experimenters or the nursing home staff was not possible.

The same research group [37] published a study protocol to investigate the effects of short-term combination (three days per four weeks) exercise training (aerobic and strength, and stretching exercise training) on different cognitive functions (executive functions, attention, episodic memory, short-term memory, working memory, reading ability, and processing speed) in healthy older people. Two parallel groups of single-blinded individuals in two parallel groups participated: training group and a waiting list control group.

Within the same perspective, a second study protocol [38] aimed to evaluate transfer of Learning Therapy consisting in reading Japanese aloud (language problems range in difficulty from 17 characters to 110 characters) and solving simple calculations (arithmetic problems difficulty range from single-digit addition to three-digit division) for 6 months, to attention and memory in healthy older adults. Participants were also required to do homework for 4-6 days a week. Both reading aloud and solving arithmetic problems require working memory, and it was expected that this prefrontal stimulation might engender a positive transfer effect in other cognitive functions. Two single-blind randomized controlled trials were carried out [39] using Learning Therapy with 32 indi-viduals affected by senile de-mentia of the Alzheimer type recruited from a nursing home and 124 participants with geriatric depression. In both investigations, the daily training program for the intervention groups continued for approximately 5 days a week for six months. A transfer effect provided some evidence that cognitive training maintains the cognitive functions of dementia patients and healthy seniors. MMSE score did not show any significant changes between baseline and follow-up measurements in the first study while the MMSE score for control subjects showed a signifi-cant decrease. In the second investigation, the MMSE score of the experimen-tal group was higher than that of the control group. However, this difference was not significant. The impact of a brain training game (Brain Age) on a wide range of cognitive functions in young adults and in elderly people was also confirmed in two double blind randomized controlled trials [40-41]. Therefore, commercial brain training game might be a simple and convenient means to improve some cognitive functions.

\subsection{Compensatory approaches}

The main aim of memory rehabilitation is to make participants as autonomous as possible in everyday functioning. Compensatory Cognitive Rehabilitation (CCR), supplies external and prosthetic guidance for disorders [42]. Consequently, systematic exercise by using external memory aids is the most typical and useful method to compensate for prospective memory deficits. 
Nevertheless, knowing when and how to employ an external aid may cause problems for subjects who have memory deficits in that using an aid is already a memory task. Evans et al. [43] indicated the best predictors of the efficacy of memory aids (e.g. calendars, electronic systems and other devices) in subjects with traumatic brain injury:

a) Current age (the younger the individuals, the more likely they will employ memory aids);

b) Period of time since traumatic event (if the temporal extension from injury is greater, the likelihood of using memory aids is reduced);

c) Number of external strategies in use antecedent to trauma.

At the outcome level, all studies on external memory aids indicated an improvement in memory function minimizing everyday failures also for patients affected by neuro-degenerative diseases. Specifically, the effectiveness of compensatory strategies varies according to the types of aids and types of patients. Using a commercial smart-phone, Svoboda and Richards [44] demonstrated the potentiality of the training program in encouraging a person with relatively serious memory deficits. Patients demonstrated maintenance and generalization of acquired smart-phone skills to several real-life memory tasks indicating that as result of systematic training persons with severe memory problems [45] can show an enhanced self-confidence in coping with memory demanding situations.

\subsection{Non-invasive brain stimulation}

Episodic memory, encoding and retrieval activities present distinct neural networks. Lesion and functional imaging observations have suggested that episodic memory pertains a distributed network of brain areas, including the PFC and PARCs. In aging, successfully encoding and retrieval mechanisms depend from the activation of both left inferior parietal lobules (IPLs) and anterior hippocampus. Combining fMRI and rTMS, Manenti and colleagues [46] provided evidence for an active involvement during retrieval not only for the PFC but for PARCs too. The employment of non-invasive brain stimulation to investigate cognitive functioning in strokes, TBI and neuro-degenerative conditions has attracted growing interest. In particular, rTMS has been demonstrated to temporarily alter neural excitability showing that the modulatory effects of cortical stimulation may endure following the immediate stimulation period. Transcranic magnetic stimulation firstly concerned a single and powerful (shorter than $1 \mathrm{~ms}$, about $2 \mathrm{~T}$ ) magnetic pulse to the scalp with a coil inducing a transitory electric flow in the cortical area below the coil, which induces a depolarization of cell membranes and transynaptic depolarization of an aggregation of cortical neurons.

Recently, progresses in technology enable the administration of magnetic pulses in trains with a rhythmic repetition rate of up to $100 \mathrm{~Hz}$ : repetitive transcranic magnetic stimulation (rTMS) appears to interact with cortical activation in a repetitive frequency-dependent mode. Several observations have suggested that continuous rTMS (lower $1 \mathrm{~Hz}$ ) decreases cortical excitability, and intermittent rTMS trains (higher up $5 \mathrm{~Hz}$ ) has the reverse effect [47]. The potential of applications for developing new therapeutic approaches is intriguing for clinical researchers. The opportunity to merge cognitive rehabilitation treatment with non-invasive brain stimulation seems to increase the effectiveness of intervention in multidimensional intervention paradigms targeted at persons with memory deficits. In the future further studies will be needed to determine the best responders to particular non-invasive brain stimulation techniques. Different fields of research need to assess what functional alterations in cortical reactivity and connectivity occurred before, during and after intervention as a consequence of cognitive plasticity.

\subsection{The ultimate strategy for memory rehabilitation: neuro-feedback training.}

EEG biofeedback represents the most recent conceptualization of the rehabilitation approach [48]. Most research suggests that significant improvements occur 75 to $80 \%$ of the time. These techniques employ operant conditioning paradigms of brainwaves, which change their frequency patterns with reinforcement. The participant has to alter the amplitude, frequency or coherence of her/his brain's electric activity and learn how to effect it. The purpose is to train the subject to normalize irregular EEG patterns returning to normative, pre-existing functioning. There are four innovative forms of neuro-feedback: 1) the Flexyx device, 2) the standard quantitative EEG (SQ), 3) the closed eye? QEEG database (EcQ), 4) activation database QEEG (ActQ). 
In order to decrease EEG amplitudes, the first device supplies a very low level of electromagnetic stimulation based on the dominant EEG pattern. Typical QEEG-guided (SQ) biofeedback, applied to individuals with ADHD or other learning disabilities, is addressed to improve beta patterns (13$20 \mathrm{~Hz}$ ) and diminish theta patterns $(4-8 \mathrm{~Hz})$ on the sensorimotor strip. A more recent third method compares the subject's closed eye QEEG with a normative database (EcQ). The activation database QEEG-guided biofeedback (ActQ) is the result of more recent progress in electroencephalography technology and allows assessment of the subjects' brain activity when they are engaged in cognitive tasks [49]. The QEEG parameters will then be compared to the normative database scores for attention, memory or problem solving.

In the last few years, a fascinating advancement has been the use of fMRI as neuro-feedback with the advantage of examining the functioning of deep subcortical areas of the brain. The serious disadvantage of fMRI neuro-feedback is that it is very expensive. A review summarizes the findings resulting in cognitive functioning after differential intervention programs; in specific computer interventions, cognitive strategies, neuro-feedback, and pharmacological therapy for TBI are compared [49]. The effect size (ES) of the treatment outcomes used in order to evaluate the effectiveness of QEEG database guided biofeedback in the rehabilitation of brain functions suggests a particular efficacy of this method on participants suffering from traumatic brain injury [11]. Specifically, in this study memory improvement could be attributed to the decrease of theta patterns that have an effect on the cellular action of memory through its role in increasing longterm potentiation and recognition performance, and the increase of upper alpha rhythm (9.5-12 $\mathrm{Hz}$ ) reflecting retrieval mechanism from long-term memory [11].

\section{Conclusions}

Memory dysfunction is a main cause of disability for individual with brain injury or other forms of degenerative disorders because even mild memory impairments can significantly inhibit the accomplishment of basic daily or return to work and represents a major obstacle to improving quality of life. The main aim of the present review was to address the question of whether memory rehabilitation can be effective, considering which types of patients would preferably benefit from specific interventions.

The reviewed studies usually support the practice of memory rehabilitation to improve memory in specific tasks, but lack of satisfactory evidence to establish the effects of memory training on relevant functional outcomes. In particular, internal and restorative strategies are recommended as a practice guideline for individuals with memory impairments due to a stable brain disorders but not for individuals with progressive cerebral disorders. The effectiveness of compensatory strategies can be suggested for a great number of pathological conditions but the degree and nature of persistent memory deficits vary among people with memory problems, and program efficacy might depend from many factors. There may be also inherent differences between rehabilitation studies using patients drawn from clinical settings versus community-based recruitment efforts; a possibility that should be further examined in future research. Neuro-feedback techniques may be more sensitive in the assessment of structural and functional abnormalities and demonstrated long-term effects. The QEEG-guided biofeedback approach appears to be the most promising to obtain meaningful results.

\section{REFERENCES}

[1] Gabrieli, J. D. E. Cognitive neuroscience of human memory. Annu. Rev. Psychol., 1998, 49, 87-115.

[2] Ptak, R., Van der Linden, M., Schnider, A. Cognitive rehabilitation of episodic memory disorders: from theory to practice. Front in Hum Neuros, 2010, 4, Article 57. doi: 10.3389/fnhum.2010.00057. 
[3] Tulving,E. Elements of Episodic Memory. London: Oxford University Press, 1983.

[4] Tulving, E., Kapur,S., Craik, F.I.M., Moscovitch, M., Houle, S. Hemispheric encoding/retrieval asymmetry in episodic memory: positron emission tomography findings. Proc.Natl.Acad.Sci. U.S.A. 1994, 91, 1989-1991.

[5] Cabeza, R. Hemispheric asymmetry reduction in older adults: the HAROLD model. Psychol.Aging, 2002, 17, 85-100.

[6] Rossi, S., Miniussi,C., Pasqualetti, P., Babiloni, C., Rossini, P.M., Cappa, S.F. Age-related functional changes of pre-frontal cortex in long-term memory: a repetitive transcranial magnetic stimulation study. J. Neurosci, 2004, 24, 7939-7944.

[7] Cotelli, M., Manenti, R., Zanetti, O., Miniussi, C. Non pharmacological intervention for memory decline (2012). Frontiers in Human Neuroscience, 2012, 6, Article 46. doi: 10.3389/fnhum.2012.00046.

[8] Piras, F., Borella, E., Incoccia, C., Carlesimo, G.A. Evidence-based practice recommendations for memory rehabilitation. Eur $\mathrm{J}$ of Phys and Rehab Med, 2011, 47, 149-175.

[9] Clare, L., Jones, R. S. P. Errorless learning in the rehabilitation of memory impairment: a critical review. Neuropsychol. Rev. 2008, 18, 1-23.

[10] Wilson, B. Memory Rehabilitation. Integrating Theory and Practice. New York: The Guilford Press, 2009.

[11] Thornton, K.E., Carmody, D.P. Efficacy of Traumatic Brain Injury rehabilitation: Interventions of QEEG-guided Biofeedback, computers, strategies, and medications Applied Psychophysiol Biofeed, 2008, 33, 101-124. doi: 10.1007/s10484008-9056-z.

[12] Rapp, S., Brenes, G., Marsh, A.P. Memory enhancement training for older adults with mild cognitive impairment: a preliminary study. Aging Ment. Health, 2002, $6,5-11$.

[13] Wilson, B. Management and remediation of memory problems in braininjured adults. In: Baddeley A.D., Kopelman M.D., Wilson B.A, (Eds). The handbook of memory disorders. 2nd edn. Chichester: Wiley, p. 617-636, 2002.

[14] das Nair R., Lincoln N. B. Evaluation of rehabilitation of memory in neurological disabilities (ReMiND): A randomized controlled trial. Clin Rehab., 2012, 26, 894-903.

[1] Hampstead, B.M., Gillis, M.M., Stringer, A.Y. Cognitive rehabilitation of memory for mild cognitive impairment: A methodological review and model for future research. $J$ of the Intern Neuropsych Soc, 2014, 1, 1-17. doi: 10.1017/S1355617713001306.

[2] das Nair et al. Rehabilitation of memory following Brain Injury (ReMemBrln): Study protocol for a randomised controlled trial. Trials, 2015, 16, 6.

[1] O'Neill Pirozzi T., et al., A controlled treatment study of internal memory strategies (I-MEMS) following traumatic brain injury. J Head Trauma Rehabil 2010, 25, 43-51.

[1] Petersen, R.C. Mild cognitive impairment as a diagnostic entity. J. Intern. Med. 2004, 256, 183-194. 
[2] Morris, J.C., et al., Harmonized diagnostic criteria for Alzheimer's disease: recommendations. J of Intern Med, 2014, 275, 204-213.

[3] Suzuki et al. Effects of multicomponent exercise on cognitive function in older adults with amnestic mild cognitive impairment: A randomized controlled trial. BMC Neurology, 2012, 12, 128. doi: 10.1186/1471-2377-12-128

[1] Pino, O., Poletti, F. Caffarra, P. Cognitive demand and reminders effect on time-based prospective memory in amnesic mild cognitive impairment (AMCl) and in healthy elderly, Open Journal of Medical Psychology, 2013, 2, 35-46. doi: 10.4236/ojmp.2013.21007.

[2] Hampstead, B.M., et al., Activation and effective connectivity changes following explicit-memory training for face-name pairs in patients with mild cognitive impairment: A pilot study. Neurorehabil. Neural Repair, 2011, 25, 210-222.

[3] Cipriani, G., Bianchetti, A., Trabucchi, M. Outcomes of a computer-based cognitive rehabilitation program on Alzheimer's disease patients compared with those on patients affected by mild cognitive impairment. Arch.Gerontol. Geriatr. 2006, 43, 327-335.

[4] McDaniel M.A., Einstein, G. O. Prospective memory: an overview and synthesis of an emerging field. Los Angeles: Sage, 2007.

[5] Shum D, Fleming, J. M., Neulinger, K. Prospective memory and traumatic brain injury: A review. Brain Impair, 2002, 3, 1-16.

[6] Pino, O., Moderato, P. (1996). II deterioramento nella memoria nell'invecchiamento: un confronto tra soggetti sani e soggetti con malattia di Alzheimer. In P. Giordano, A. Svettini \& G. Bonaviri (Eds.), L'invecchiamento tra normalità e patologia, Atti delle VII Giornate psichiatriche in Lampedusa. Agrigento: Editrice Etnea, pp. 361-373, 1996.

[7] Baddeley, A., Wilson, B.A. When implicit learning fail: Amnesia and the problem of error elimination. Neuropsych, 1994, 32, 53-68.

[8] Glisky, E. L., Schacter, D. L., Tulving, E. Computer learning by memory impaired patients: Acquisition and retention of complex knowledge. Neuropsych, 1986, 24, 313-328.

[9] Glisky, E. L., Schacter, D. L. Extending the limits of complex learning in organic amnesia: computer training in a vocational domain. Neuropsych, 1989, 27, 107120.

[10] Leng, N. R. C., Parkin, A. J. Amnesic patients can benefit from instructions to use imagery: Evidence against the cognitive mediation hypothesis. Cortex, 1988, 24, 33-39.

[11] Schacter, D. L., Rich, S. A., Stampp, M. S. Remediation of memory disorders: experimental evaluation of the spaced retrieval technique. J. Clin. Exp. Neuropsychol. 1985, 7, 79-96.

[12] Ebbinghaus, H.. Über das Gedächtnis. Untersuchungen zur experimentellen Psychologie. Darmstadt: Wissenschaftliche Buchgesellschaf, 1885/1992.

[13] Sohlberg, M. M., Mateer, C. A. Introduction to cognitive rehabilitation. New York: Guilford Press, 1989.

[14] Pino, O., Gentile, R. Procedure per controllare l'overselectivity nell'autismo infantile: Ricerca sperimentale. Ter del Comp, 1990, II, 157-161. 
[15] Malane, M.L., Skrainer, M.J., Camp, C.J., Neundorfer, M., Garzelle, G.J. Research in practice II: Spaced retrieval. A memory intervention. Alzh Care Quart, 2007, 8, 65-74.

[16] Kawashima R, et al., Reading aloud and arithmetic calculation improve frontal function of people with dementia. J Gerontol A Biol Sci Med Sci 2005, 60, 380384.

[17] Nouchi, R. et al. Beneficial effects of short-term combination exercise training on diverse cognitive functions in healthy older people: study protocol for a randomized controlled trial. Trials, 2012a, 13, 200. doi: 10.1186/1745-6215-13-200.

[1] Nouchi et al. Beneficial effects of reading aloud and solving simple arithmetic calculations (learning therapy) on a wide range of cognitive functions in the healthy elderly: study protocol for a randomized controlled trial. Trials, 2012b, 13, 32. doi: 10.1186/1745-6215-13-32.

[1] Kawashima, R. Mental exercises for cognitive function: Clinical evidence. J Prev Med Public Health, 2013, 46, S22-S27 http://dx.doi.org/10.3961/jpmph.2013.46.S.S22.

[1] Nouchi R., et al. Brain Training Game Boosts Executive Functions, Working Memory and Processing Speed in the Young Adults: A Randomized Controlled Trial. PLoS ONE, 2013, 8: e55518. doi:10.1371/journal.pone.0055518.

[1] Nouchi, R. et al. Four weeks of combination exercise training improved executive functions, episodic memory, and processing speed in healthy elderly people: evidence from a randomized controlled trial. Age, 2014, 36, 787-799. doi: 10.1007/s11357-013-9588-X.

[2] Wehman, P., Kreutzer, J., Sale, P., West, M., Morton, M., Diambra, J. Cognitive impairment and remediation: Implications for employment following traumatic brain injury. The $J$ of Head Traum Reh, 1989, 4, 66-75.

[1] Evans, J., Wilson, B., Needham, P., Brentnall, S. Who makes good use of memory aids? Results of a survey of people with acquired brain injury. J. Int. Neuropsychol. Soc. 2003, 9, 922-935.

[2] Svoboda, E., Richards, B. Compensating for anterograde amnesia: A new training method that capitalizes on emerging smartphone technologies. J. Int. Neuropsychol. Soc. 2009, 15, 629-638.

[3] Svoboda, E., Richards, B., Polsinelli, A., Guger, S. A theory-driven training programme in the use of emerging commercial technology: Application to an adolescent with severe memory impairment. Neuropsychol. Rehabil., 2010, 1-25. doi: 10.1080/09602011003669918.

[4] Manenti, R.,Tettamanti, M.,Cotelli, M., Miniussi, C., Cappa, S.F. The neural bases of word encoding and retrieval: afMRI-guided transcranial magnetic stimulation study. Brain Topogr., 2010, 22, 318-332.

[5] Pascual-Leone, A., Valls-Sole, J., Wassermann, E.M., Hallett, M. Responses to rapid rate transcranial magnetic stimulation of the human motor cortex. Brain, 1994, 117 (Pt 4), 847-858.

[6] Hammond, D. C. What is neurofeedback: an update. Journal of neurotherapy: Investigations in neuromodulation, Neurof and Appl Neurosc., 2011, 15, 4, 305-336. doi: 10.1080/19874208.2011.623090. 
[7] Rajakumari, P.R., Jamuna, N., Indira Devi, B., Thennarasu K.M. Neurofeedback training to enhance learning and memory in patients with traumatic brain injury: A single case study. Ind J of Neurotr (IJNT), 2009, 6, 87-90. 